\title{
Epialleles in plant evolution
}

\author{
Detlef Weigel ${ }^{1, *}$ and Vincent Colot $2,3,4, * *$
}

\begin{abstract}
Heritable phenotypic differences caused by epigenetic modifications, rather than DNA sequence mutations, pose a challenge to our understanding of natural variation. Here, we review what is known about plant epialleles and the role of epigenetics in evolution.
\end{abstract}

Keywords DNA methylation, epialleles, epigenetics, genetics, plant evolution

\section{Introduction}

In college, biologists learn how Darwin and Mendel, whose ideas eventually resulted in the modern evolutionary synthesis [1], prevailed over Lamarck and Lysenko. Now, from a cursory reading of the literature, it is possible to get the impression that this is changing [2-4]. But, although epigenetics is clearly enriching modern genetic research, reports of the end of genetics have - in our opinion - been an exaggeration. Since the 1990s, the molecular basis of hundreds of naturally occurring phenotypic variants has been identified in crop or wild species and, overwhelmingly, DNA sequence differences are involved (for example, [5-7]). Indeed, the number of natural epialleles that we know of in plants is only about a dozen. However, it is very likely that there is an ascertainment bias in favor of DNA sequence changes in the studies carried out so far. Thus, the extent to which epigenetic variation contributes to phenotypic variation in plants is still not known with certainty.

Epigenetics was a term coined by Waddington, to reflect - in modern terms - the causal mechanisms that lie between genes and phenotypes [8]; however, today it is mainly used to describe modifications that cause changes in gene expression that are stably transmitted during mitosis or meiosis, but that do not involve

*Correspondence: weigel@tue.mpg.de, colot@biologie.ens.fr

'Department of Molecular Biology, Max Planck Institute for Developmental Biology, Tübingen, 72076, Germany

¿Ecole Normale Supérieure, Institut de Biologie de l'ENS (IBENS), Paris, F-75005, France

Full list of author information is available at the end of the article differences in the underlying DNA sequence. In eukaryotes, most known epigenetic mechanisms are chromatin based, and may involve still poorly defined combinations of post-translational histone modifications and histone variants, small or long non-coding RNAs, and DNA methylation [9]. Although the role of epigenetic processes in development is now well established, the field that investigates the transgenerational inheritance of epigenetic modifications is still in its infancy.

\section{Natural epialleles in plants}

The first natural plant mutant for which the molecular basis was determined to be an epimutation rather than a change in DNA sequence was a peloric variant of toadflax, Linaria vulgaris. Whereas flax normally has bilaterally symmetrical flowers, the flowers of this variant show radial symmetry, a phenotype strikingly similar to the one seen in induced cycloidea (cyc) mutants of snapdragon, Antirrhinum majus [10]. Isolation of a cyc homolog from flax revealed that it was genetically linked to the peloric phenotype, and that RNA of the $c y c$ homolog did not accumulate in the peloric strain [11]. The open reading frame of $c y c$, however, appeared intact, and on DNA blots no gross differences could be detected around the gene. In contrast, analysis with DNA-methylation-sensitive restriction enzymes provided evidence for increased methylation of the cyc locus. A direct relationship between DNA methylation and reduced $c y c$ expression was deduced from the analysis of spontaneous somatic revertants ranging in phenotype from semipeloric to near wild type. In these plants, DNA methylation was reduced, which also confirmed that the open reading frame was indeed intact and functional. Notably, demethylation was less pronounced in semi-peloric than in near-wild-type flowers, both supporting a direct relationship between DNA methylation and gene expression, and confirming that DNA methylation is not necessarily an all-or-nothing affair [11,12].

A second example of a natural epimutation is provided by the colorless nonripening (CNR) locus from tomato [13]. As in the example from flax the causal locus, which encodes another type of transcription factor, is intact in the non-ripening strain, but expressed at much lower levels. Again, there were differences in DNA methylation. This being a more recent study, the authors were able to 
investigate DNA methylation more quantitatively, using bisulfite sequencing. A block of about 300 bp approximately $2 \mathrm{~kb}$ upstream of the gene was heavily methylated in the non-ripening plants. Strikingly, the Liberto wildtype background, in which the colorless nonripening variant was found, was also quite highly methylated in this region, although not quite as extensively as CNR plants. In contrast, another wild-type strain, Ailsa Craig, had very low levels of DNA methylation at the locus, even though there were no obvious differences in the DNA sequence. Thus, it is possible to speculate the Liberto strain is more likely to give rise to $\mathrm{Cnr}$ mutant plants than the Alisa Craig strain.

Within $95 \mathrm{~kb}$ around the $\mathrm{Cnr}$ locus, the epimutant and the Liberto parent had no DNA sequence differences. Since the final mapping interval of $13 \mathrm{~kb}$ was approximately in the center of this $95 \mathrm{~kb}$ region, it is reasonable to assume that a nearby structural variation is not responsible for the modification of Cnr chromatin [13], although such a variant might have provided an initial trigger, as discussed below with respect to FOLT in Arabidopsis thaliana [14].

In contrast to $c y c$ and $C n r$, several other epialleles are clearly associated with alterations in DNA sequences. Because DNA methylation may spread outwards from repeats and transposable elements [15-17] - although it does not always [18] - structural variants could in fact be the primary causes of differences in the activity of adjacent genes, with DNA methylation playing a secondary or mediating role. One such example is provided by melon plants, in which the gynoecious $(g)$ locus is inactive and which therefore produce only female flowers [19]. The sequences responsible for loss of $g$ expression were mapped to a 1.4-kb non-coding sequence, which contained a DNA transposon insertion in all gynoecious plants tested. The transposon was heavily methylated, and high level DNA methylation was also detected in the promoter of the adjacent gene, perhaps as a result of spreading from the transposon. However, in a recombinant gene in which the transposon was segregated away, DNA methylation was reduced and gene expression was increased. Moreover, DNA methylation was much lower in phenotypically revertant branches, indicating that the transposon effects were variable, at least to a certain extent. A similar case has been described for rice plants with a metastable epiallele at the DWARF1 (D1) locus [20], with a large tandem repeat being responsible for variable DNA methylation.

Thus, all natural epialleles reported to date and for which sequence information is available, have involved a gain or loss of DNA methylation. Moreover, these differences in DNA methylation are often in transposable elements or other types of repeat sequences located near or within the affected genes. This suggests that the 'epimutability' of many genes is ultimately conditioned by the presence of repeat sequences near or within them, and is thus likely to differ substantially between genotypes (Figure 1a).

\section{Communication between homologous sequences}

In $A$. thaliana, tandem repeats in the promoter are also associated with gene silencing, in this case of the FWA gene. In wild-type plants, these repeats are methylated, except in the triploid endosperm, where the two copies of the maternal allele are demethylated and expressed [21]. Stable epialleles in which the repeats have become unmethylated throughout the life cycle have been obtained either after ethyl methanesulfonate (EMS) mutagenesis, or in plants that are defective in DNA methylation. Demethylation in the adult plant leads to activation of $F W A$ and late flowering [22,23]. Once fully unmethylated, these tandem repeats very rarely, if ever, become spontaneously re-methylated [22,24]. In contrast, when an unmethylated copy is transformed into wildtype plants, its repeats become rapidly methylated, shutting down expression of the transgene, apparently because of information transfer from the endogenous, methylated copies [25]. Such a communication between alleles may be widespread [26], but is not observed in crosses of plants with a methylated and silenced FWA allele to plants with an unmethylated, activated copy at the endogenous locus, and fwa epimutants therefore behave like normal mutants (as do the examples discussed above, with the exception of the reversion events).

Epigenetic interactions at the FWA locus thus differ from the classic examples of paramutation in maize, in which silenced alleles frequently induce silencing of normal alleles [27,28] (Figure 1). Nonetheless, as FWA, paramutation has been linked to tandem repeats in the promoter of paramutable alleles at the maize $b 1$ locus [29]. Tandem repeats are seemingly also important for paramutation at the $r 1$ locus, but in this case they are apparently much larger, as the $r 1$ locus is a tandem array of several very similar genes [30]. In contrast, the role of repeats in paramutation at the $p 1$ locus is less clear [31].

Just as information between alleles or between endogenous genes and transgenes can be transferred (relying on short interfering RNAs (siRNAs) and the DNA methylation machinery they recruit $[28,32])$, there is communication between homologous sequences throughout the genome. The first case reported in A. thaliana was that of the $P A I$ family of genes. One natural strain of A. thaliana has two $P A I$ genes in an inverted tandem arrangement, plus two more dispersed single copies, and all four genes are heavily methylated [33]. Another strain has only three single copies, which are not methylated, but which become methylated after a cross to the strain with the inverted tandem copies $[18,34]$. 


\section{Trigger present}

(a)

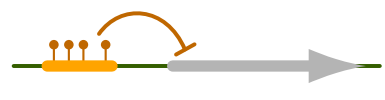

(b)

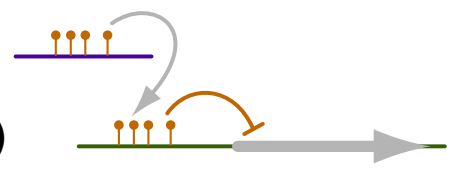

(c)

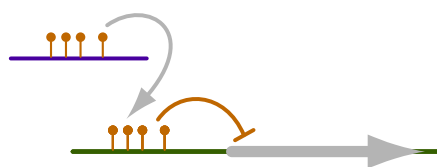

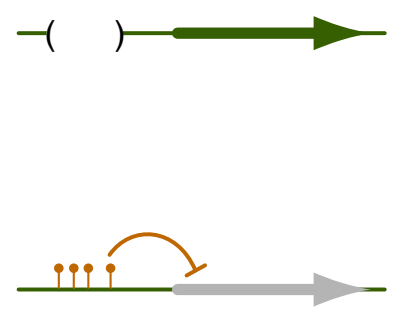

\section{Trigger absent}

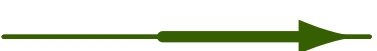

Figure 1. Classes of epialleles. (a) Epigenetic modifications and associated silencing of the adjacent gene is dependent on a specific cis-element, often a repetitive element. (b) Epigenetic modification is triggered by another locus or allele. Once the modification has been established, the trigger is no longer required for its maintenance. (c) Epigenetic modification is triggered by another locus or allele, but the trigger is permanently required.

A similar situation, with interesting phenotypic consequences, is seen at the FOLT1 and FOLT2 loci in A. thaliana [14]. In one strain, the FOLT2 locus contains multiple truncated copies, and siRNAs produced by these truncated versions target the intact FOLT1 copy and silence it. Notably, FOLT2 itself escapes complete silencing, preserving FOLT activity. Another strain lacks the FOLT2 locus, which induces silencing, but has an active FOLT1 copy. When this copy is replaced by the silenced FOLT1 allele from the other strain through crossing, plants lack FOLT activity and almost always die [14]. Important for this phenomenon is that FOLT1 stays silenced even after the FOLT2 locus that induces silencing has been segregated away (Figure 1b). In other words, FOLT1 may be seen as a 'pure' epiallele [35], but without complete information about the history of the genetic background it has passed through, it is impossible to know whether it reached this state without any external influence. Genome-wide analyses with genetic material derived from crossing closely related tomato species have recently confirmed that such trans interactions are likely to be quite common, and that they may underlie many aspects of the superior or inferior performances of hybrid plants [36]. An important finding in this case was that silencing was only established gradually similar to what has been observed in A. thaliana [37] which is discussed below. Once complete genome sequences for the tomato lines become available, it will also be possible to address systematically the question of whether there are epialleles that are absolutely dependent on a trans-acting trigger (Figure 1c).
Spontaneous changes in DNA methylation patterns

The examples discussed so far indicate that changes in DNA methylation patterns are far from random, but that they are also not always entirely predictable. To distinguish the effects of interactions between different genomes, and of new structural variants from spontaneous changes, whole-genome methylation patterns were studied in isogenic $A$. thaliana lines [38,39]. Lines were derived from a single progenitor and then propagated in a benign greenhouse environment by single-seed descent. After thirty generations, almost $10 \%$ of all methylated cytosines in the genome had increased or decreased methylation in at least one out of ten lines examined. However, there is little evidence that such differentially methylated positions (DMPs) can have major effects on the activity of adjacent genes. Rather, it is large contiguous regions of differential methylation (differentially methylated regions, or DMRs), as in the epialleles discussed above, which normally matter. In contrast to DMPs, there were very few DMRs in the studied $A$. thaliana lines [38,39].

In agreement with what is known about the establishment and maintenance of DNA methylation, DMPs were not randomly distributed. DNA methylation on and near transposons was highly stable, whereas it often changed over genes and far away from transposons. Moreover, the same changes were seen much more often than expected by chance in different lines, indicating that certain sites are considerably less stable than others. The bias in spontaneous DNA methylation changes parallels what has been reported for differences between wild strains, in 


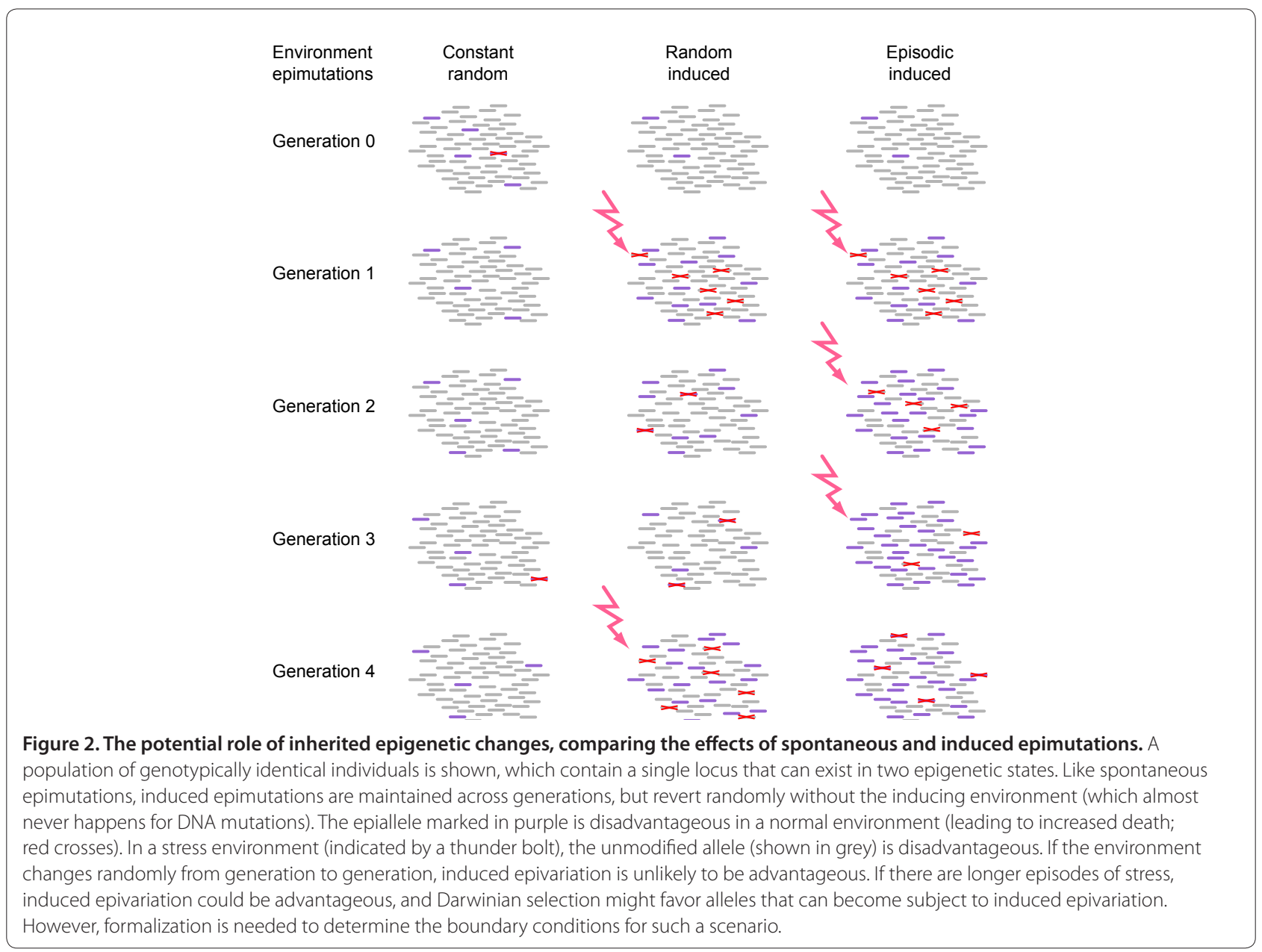

which transposon methylation is much more similar than genic methylation [40]. It is also consistent with transposon methylation being under much greater selective pressure. Loss of DNA methylation has comparatively few effects on the expression of protein-coding genes, but it greatly reduces transposon silencing [41-43]. In turn, active transposons are powerful mutagens.

\section{Lessons from crosses between methylated and demethylated genomes}

Given the frequent implication of repeat elements in the epimutability of genes, an important question is the extent to which the accidental loss of DNA methylation over transposons and other repeats can be inherited and affect phenotypes. Two experimental studies have provided genome-wide answers to this question in A. thaliana $[44,45]$. Both studies relied on the creation of epigenetic recombinant inbred lines (epiRILs). In one case [44], the epiRILS were derived from the cross of a wild-type individual with a near-isogenic plant homozygous for a mutant allele of MET1, which encodes the main DNA methyltransferase responsible for maintaining CG methylation in repeat sequences, as well as in gene bodies. In another case [45], a wild-type individual was crossed with a plant mutant for DDM1, which encodes a putative chromatin remodeler involved in maintaining all types of DNA methylation (CG, CHG and $\mathrm{CHH}$ ), specifically over repeat sequences. After the initial cross, a single $F_{1}$ individual was either selfed [44], or backcrossed to the wild-type parent [45]. $F_{2}$ progeny homozygous for the wild-type MET1 or DDM1 allele were selected, and epiRILs were propagated through seven rounds of selfing. Analysis of these lines indicated that met1- and ddm1induced hypomethylation of repeat sequences could be either stably inherited for at least eight generations or else fully reversed $[44,45]$. Reversion was mediated by small RNAs mainly acting in cis, and often occurred in several steps over successive generations [37]. Moreover, heritable variation for several complex traits was observed in the epiRILs [44-47], highlighting the potentially important role of repeat-associated epigenetic changes in generating heritable phenotypic diversity. 


\section{Outlook}

Although the mechanisms by which repeat elements are targeted for DNA methylation and become fully methylated are now understood in detail, much less is known about the tempo of this process, which presumably is both progressive over several generations and dependent on a multiplicity of factors, such as the type of repeat sequence concerned and environmental conditions. Moreover, it is still unclear how DNA methylation can be lost over repeat elements in natural settings, and how stable hypomethylation can be. Here again, the DNA sequence and environment are likely key determinants. Indeed, there are now several reports of transgenerational effects of stresses such as heat, where the progeny of stressed plants apparently withstand a specific stress better than the initial line - amazingly similar to what Lamarck and Lysenko believed [48-52]. Assuming such phenomena can be confirmed, they must be the product of Darwinian evolution, which would have produced the (epi)genetic mechanisms that underlie such transgenerational effects. That the environment can effect heritable changes is not new; inducible hypermutability is a welldocumented phenomenon in bacteria [53]. Exploring the role of the environment in inducing epigenetic variation is therefore an important task for the future, as is the study of epigenome-wide changes that can be induced by different environments. Similarly, we need more knowledge of how the genome-wide effect sizes of genetic and epigenetic alleles compare. Finally, we need an explicit theory of population epigenetics that describes the parameters under which epimutations could contribute to evolution (Figure 2).

\section{Abbreviations}

bp, base pair; kb, kilobase; DMP, differentially methylated position; DMR, differentially methylated region; epiRIL, epigenetic recombinant inbred line; siRNA, short interfering RNA.

\section{Competing interests}

The authors declare that they have no competing interests.

\section{Acknowledgments}

Our studies of epigenetic inheritance are supported by the European Union FP7 Collaborative Project AENEAS (contract KBBE-2009-226477, to DW), the European Union FP7 Network of Excellence Epigenesys (to VC) and the French Agence Nationale de la Recherche project EPIMOBILE (to VC).

\section{Author details}

'Department of Molecular Biology, Max Planck Institute for Developmental Biology, 72076, Tübingen, Germany. ${ }^{2}$ Ecole Normale Supérieure, Institut de Biologie de l'ENS (IBENS), Paris, F-75005, France. ${ }^{3}$ CNRS UMR 8197, Paris, F-75005, France. ${ }^{4}$ Inserm U1024, Paris, F-75005, France.

Published: 11 October 2012

\section{References}

1. Huxley J: Evolution: the Modern Synthesis. Cambridge: MIT Press; 2010.

2. Jablonka E, Lamb MJ: Evolution in Four Dimensions: Genetic, Epigenetic, Behavioral, and Symbolic Variation in the History of Life. Cambridge: MIT Press; 2005.

3. Grant-Downton RT, Dickinson HG: Epigenetics and its implications for plant biology. 2. The 'epigenetic epiphany': epigenetics, evolution and beyond. Ann Bot 2006, 97:11-27.

4. Maderspacher F: Lysenko rising. Curr Biol 2010, 20:R835-837.

5. Doebley JF, Gaut BS, Smith BD: The molecular genetics of crop domestication. Cell 2006, 127:1309-1321.

6. Xing Y, Zhang Q: Genetic and molecular bases of rice yield. Annu Rev Plant Biol 2010, 61:421-442

7. Weigel D: Natural variation in Arabidopsis: from molecular genetics to ecological genomics. Plant Physiol 2012, 158:2-22

8. Waddington CH: The epigenotype. Endeavour 1942, 1:18-20.

9. Bird A: Perceptions of epigenetics. Nature 2007, 447:396-398

10. Luo D, Carpenter R, Vincent C, Copsey L, Coen E: Origin of floral asymmetry in Antirrhinum. Nature 1996, 383:794-799.

11. Cubas P, Vincent C, Coen E: An epigenetic mutation responsible for natural variation in floral symmetry. Nature 1999, 401:157-161.

12. Martienssen R, Barkan A, Taylor WC, Freeling M: Somatically heritable switches in the DNA modification of Mu transposable elements monitored with a suppressible mutant in maize. Genes Dev 1990, 4:331-343.

13. Manning K, Tor M, Poole M, Hong Y, Thompson AJ, King GJ, Giovannoni JJ, Seymour GB: A naturally occurring epigenetic mutation in a gene encoding an SBP-box transcription factor inhibits tomato fruit ripening. Nat Genet 2006, 38:948-952

14. Durand S, Bouche N, Perez Strand E, Loudet O, Camilleri C: Rapid establishment of genetic incompatibility through natural epigenetic variation. Curr Bio/ 2012, 22:326-331.

15. Saze H, Kakutani T: Heritable epigenetic mutation of a transposon-flanked Arabidopsis gene due to lack of the chromatin-remodeling factor DDM1. EMBO J 2007, 26:3641-3652.

16. Cokus SJ, Feng S, Zhang X, Chen Z, Merriman B, Haudenschild CD, Pradhan S, Nelson SF, Pellegrini M, Jacobsen SE: Shotgun bisulphite sequencing of the Arabidopsis genome reveals DNA methylation patterning. Nature 2008, 452:215-219.

17. Ahmed I, Sarazin A, Bowler C, Colot V, Quesneville H: Genome-wide evidence for local DNA methylation spreading from small RNA-targeted sequences in Arabidopsis. Nucleic Acids Res 2011, 39:6919-6931.

18. Melquist S, Luff B, Bender J: Arabidopsis PAl gene arrangements, cytosine methylation and expression. Genetics 1999, 153:401-413.

19. Martin A, Troadec C, Boualem A, Rajab M, Fernandez R, Morin H, Pitrat M, Dogimont $C$, Bendahmane A: A transposon-induced epigenetic change leads to sex determination in melon. Nature 2009, 461:1135-1138.

20. Miura K, Agetsuma M, Kitano H, Yoshimura A, Matsuoka M, Jacobsen SE, Ashikari M: A metastable DWARF1 epigenetic mutant affecting plant stature in rice. Proc Natl Acad Sci U S A 2009, 106:11218-11223.

21. Kinoshita Y, Saze H, Kinoshita T, Miura A, Soppe WJ, Koornneef M, Kakutani T: Control of FWA gene silencing in Arabidopsis thaliana by SINE-related direct repeats. Plant J 2007, 49:38-45.

22. Kakutani T: Genetic characterization of late-flowering traits induced by DNA hypomethylation mutation in Arabidopsis thaliana. Plant J 1997, 12:1447-1451.

23. Soppe WJ, Jacobsen SE, Alonso-Blanco C, Jackson JP, Kakutani T, Koornneef M, Peeters AJ: The late flowering phenotype of fwa mutants is caused by gain-of-function epigenetic alleles of a homeodomain gene. Mo/ Cell 2000, 6:791-802.

24. Johannes F, Porcher E, Teixeira FK, Saliba-Colombani V, Simon M, Agier N, Bulski A, Albuisson J, Heredia F, Audigier P, Bouchez D, Dillmann C, Guerche P, Hospital F, Colot V: Assessing the impact of transgenerational epigenetic variation on complex traits. PLoS Genet 2009, 5:e1000530.

25. Chan SW, Zhang X, Bernatavichute YV, Jacobsen SE: Two-step recruitment of RNA-directed DNA methylation to tandem repeats. PLOS Bio/ 2006, 4:e363.

26. Greaves IK, Groszmann M, Ying H, Taylor JM, Peacock WJ, Dennis ES: Trans chromosomal methylation in Arabidopsis hybrids. Proc Natl Acad Sci U S A 2012, 109:3570-3575

27. Hollick JB: Paramutation and development. Annu Rev Cell Dev Bio/ 2010, 26:557-579.

28. Teixeira FK, Colot V: Repeat elements and the Arabidopsis DNA methylation landscape. Heredity 2010, 105:14-23.

29. Stam M, Belele C, Dorweiler JE, Chandler VL: Differential chromatin structure within a tandem array $100 \mathrm{~kb}$ upstream of the maize $b 1$ locus is associated with paramutation. Genes Dev 2002, 16:1906-1918.

30. Eggleston WB, Alleman M, Kermicle JL: Molecular organization and germinal instability of $R$-stippled maize. Genetics 1995, 141:347-360. 
31. Sidorenko $L$, Chandler V: RNA-dependent RNA polymerase is required for enhancer-mediated transcriptional silencing associated with paramutation at the maize $p 1$ gene. Genetics 2008, 180:1983-1993.

32. Law JA, Jacobsen SE: Establishing, maintaining and modifying DNA methylation patterns in plants and animals. Nat Rev Genet 2010, 11:204-220

33. Bender J, Fink GR: Epigenetic control of an endogenous gene family is revealed by a novel blue fluorescent mutant of Arabidopsis. Cell 1995, 83:725-734

34. Luff B, Pawlowski L, Bender J: An inverted repeat triggers cytosine methylation of identical sequences in Arabidopsis. Mol Cell 1999, 3:505-511.

35. Richards EJ: Inherited epigenetic variation--revisiting soft inheritance. Nat Rev Genet 2006, 7:395-401.

36. Shivaprasad PV, Dunn RM, Santos BA, Bassett A, Baulcombe DC: Extraordinary transgressive phenotypes of hybrid tomato are influenced by epigenetics and small silencing RNAs. EMBO J 2011, 31:257-266.

37. Teixeira FK, Heredia F, Sarazin A, Roudier F, Boccara M, Ciaudo C, Cruaud C Poulain J, Berdasco M, Fraga MF, Voinnet O, Wincker P, Esteller M, Colot V: A role for RNAi in the selective correction of DNA methylation defects. Science 2009, 323:1600-1604.

38. Becker C, Hagmann J, Müller J, Koenig D, Stegle O, Borgwardt K, Weigel D: Spontaneous epigenetic variation in the Arabidopsis thaliana methylome. Nature 2011, 480:245-249.

39. Schmitz RJ, Schultz MD, Lewsey MG, O'Malley RC, Urich MA, Libiger O, Schork NJ, Ecker JR: Transgenerational epigenetic instability is a source of novel methylation variants. Science 2011, 334:369-373.

40. Vaughn MW, Tanurdzic M, Lippman Z, Jiang H, Carrasquillo R, Rabinowicz PD, Dedhia N, McCombie WR, Agier N, Bulski A, Colot V, Doerge RW, Martienssen RA: Epigenetic natural variation in Arabidopsis thaliana. PLoS Biol 2007, $5: e 174$.

41. Lister R, O'Malley RC, Tonti-Filippini J, Gregory BD, Berry CC, Millar AH, Ecker $J R$ : Highly integrated single-base resolution maps of the epigenome in Arabidopsis. Cell 2008, 133:523-536.

42. Zhang X, Yazaki J, Sundaresan A, Cokus S, Chan SW, Chen H, Henderson IR, Shinn P, Pellegrini M, Jacobsen SE, Ecker JR: Genome-wide high-resolution mapping and functional analysis of DNA methylation in Arabidopsis. Cell 2006, 126:1189-1201.

43. Lippman Z, Gendrel AV, Black M, Vaughn MW, Dedhia N, McCombie WR, Lavine K, Mittal V, May B, Kasschau KD, Carrington JC, Doerge RW, Colot V,
Martienssen R: Role of transposable elements in heterochromatin and epigenetic control. Nature 2004, 430:471-476.

44. Reinders J, Wulff BB, Mirouze M, Mari-Ordonez A, Dapp M, Rozhon W, Bucher E, Theiler G, Paszkowski J: Compromised stability of DNA methylation and transposon immobilization in mosaic Arabidopsis epigenomes. Genes Dev 2009, 23:939-950.

45. Johannes F, Colomé-Tatché M: Quantitative epigenetics through epigenomic perturbation of isogenic lines. Genetics 2011, 188:215-227.

46. Roux F, Colomé-Tatché M, Edelist C, Wardenaar R, Guerche P, Hospital F, Colot $V$, Jansen RC, Johannes F: Genome-wide epigenetic perturbation jumpstarts patterns of heritable variation found in nature. Genetics 2011, 188:1015-1017.

47. Latzel V, Zhang Y, Karlsson Moritz K, Fischer M, Bossdorf O: Epigenetic variation in plant responses to defence hormones. Ann Bot 2012. doi: 10.1093/aob/mcs088.

48. Slaughter A, Daniel X, Flors V, Luna E, Hohn B, Mauch-Mani B: Descendants of primed Arabidopsis plants exhibit resistance to biotic stress. Plant Physiol 2012, 158:835-843

49. Luna E, Bruce TJ, Roberts MR, Flors V, Ton J: Next-generation systemic acquired resistance. Plant Physiol 2012, 158:844-853.

50. Holeski LM: Within and between generation phenotypic plasticity in trichome density of Mimulus guttatus. J Evol Biol 2007, 20:2092-2100.

51. Verhoeven KJF, Jansen JJ, Van Dijk PJ, Biere A: Stress-induced DNA methylation changes and their heritability in asexual dandelions. New Phytol 2010, 185:1108-1118.

52. Whittle CA, Otto SP, Johnston MO, Krochko JE: Adaptive epigenetic memory of ancestral temperature regime in Arabidopsis thaliana. Botany 2009, 87:650-657.

53. Galhardo RS, Hastings PJ, Rosenberg SM: Mutation as a stress response and the regulation of evolvability. Crit Rev Biochem Mol Biol 2007, 42:399-435.

doi:10.1186/gb-2012-13-10-249

Cite this article as: Weigel $\mathrm{D}$, Colot V: Epialleles in plant evolution. Genome Biology 2012, 13:249. 